\section{Embryonic factor helps tumours to lie low}

Cf

DUX4 tran-

scriptional

activity

strongly

correlated

with response

to anti-CTLA4

therapy

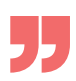

Immune checkpoint blockade in cancer relies on efficient tumour antigen presentation to activate antitumour immunity. Chew, Campbell et al. show that the early embryonic transcription factor double homeobox protein 4 (DUX4) is re-expressed in a range of cancers, and is involved in immune suppression by reducing MHC class I protein expression.

The researchers screened for cancer-specific genes by comparing the transcriptomes of 9,759 cancer samples from 33 different cancers to the transcriptomes of 34 peritumoural normal tissues or somatic tissues of healthy individuals. Ranking of the most cancer-specific genes showed that cancer-testis antigens (CTAs) were among the top ranked genes, and transcriptional regulation was the most enriched biological pathway in a gene ontology-based comparison of the highest scoring genes preferentially expressed in cancer. Of three genes with the strongest pan-cancer expression profiles, DUX4 was chosen for further analysis because of its reported implications in immunogenicity.

For activation of target genes, many of which encode CTAs, DUX4 requires the amino-terminal and carboxy-terminal domains. The researchers confirmed the expression of full length DUX4 mRNA in solid cancers at levels comparable to its endogenous expression during embryogenesis. Expression of non-functional transcripts of DUX4, including shorter splice variants or

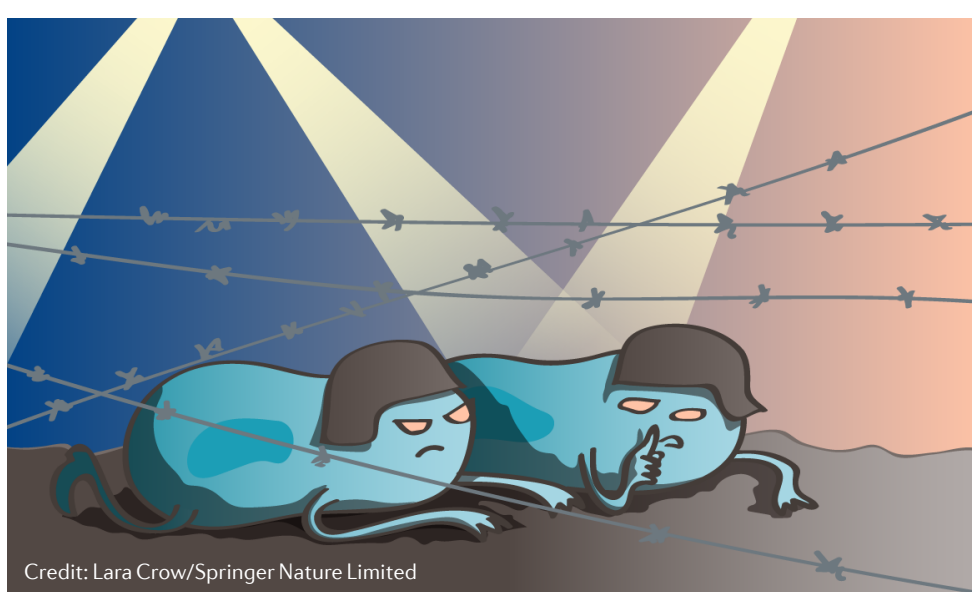

doxycycline (dox)-inducible DUX4 expression (MB135iDUX4 cells), IFN $\gamma$ increased MHC class I protein expression, which was blocked by induction of DUX4. Similarly, a range of cancer cell lines, including breast, cervical, rhabdoid and testicular cancer and melanoma, expressing the dox-inducible DUX4 construct increased MHC class I protein expression in response to IFN $\gamma$, which could be blocked by dox treatment. Moreover, induction of DUX4 in HeLa cells or MB135iDUX4 cells reduced IFN $\gamma$-mediated MHC class I expression on the cell surface (which is indicative of effective antigen binding and presentation). By contrast, dox treatment in the absence of the dox-inducible DUX4 construct did not alter the increased MHC class I protein expression in response to IFN $\gamma$ or change MHC class I expression on the cell surface in non-transformed or transformed cell lines.

If DUX4 suppressed antigen presentation in tumours, this could promote resistance to immune checkpoint blockade therapy. To test this hypothesis, the authors analysed published RNA-seq data of biopsy samples from patients with metastatic melanoma before and after treatment with anti-cytotoxic T lymphocyte antigen 4 (CTLA4). DUX4 transcriptional activity strongly correlated with response to antiCTLA4 therapy - biopsy samples from patients with non-responsive disease showed higher levels of DUX4 and target gene expression than biopsy samples from patients with responsive disease. Increased DUX4 transcriptional activity was also associated with reduced progression-free and overall survival after therapy. Similar trends were observed in biopsies from patients treated with anti-programmed cell death protein 1 ligand 1 (PDL1).

Highlighting a potential pan-cancer role for DUX4 in mediating immune evasion, this study provides a basis for exploring inhibition of DUX4 to overcome resistance to immune checkpoint blockade.

Ulrike Harjes

ORIGINAL ARTICLE Chew, G. et al. DUX4 suppresses MHC class I to promote cancer immune evasion and resistance to checkpoint blockade. Dev. Cell https://doi.org/10.1016/j.devcel.2019. 06.011 (2019) 